\title{
Dynamic Time Warping of Pulse Wave Curves
}

Alexandru-Gabriel Pielmuş, Chair of Electronics and Medical Signal Processing, Technische Universität Berlin, EMail: a.pielmus@tu-berlin.de

Dennis Osterland, Chair of Electronics and Medical Signal Processing, Technische Universität Berlin, E-Mail: info@emsp.tu-berlin.de

Timo Tigges, Chair of Electronics and Medical Signal Processing, Technische Universität Berlin, E-Mail: info@emsp.tu-berlin.de

Michael Klum, Chair of Electronics and Medical Signal Processing, Technische Universität Berlin, E-Mail: info@emsp.tu-berlin.de

Reinhold Orglmeister, Chair of Electronics and Medical Signal Processing, Technische Universität Berlin, E-Mail: info@emsp.tu-berlin.de

Aarne Feldheiser, Klinik für Anästhesiologie m.S. operative Intensivmedizin, Charité Universitätsmedizin Berlin, EMail: aarne.feldheiser@charite.de

Oliver Hunsicker, Klinik für Anästhesiologie m.S. operative Intensivmedizin, Charité Universitätsmedizin Berlin, EMail: oliver.hunsicker@charite.de

Being able to non-obtrusively and continuously monitor arterial blood pressure is of great interest, particularly in the context of wearable sensors. A common limitation is the need for dedicated hardware, which is either obtrusive or expensive. In our current work, we investigate unimodal pulse waves from three handily accessible heterogeneous sources: photoplethysmography, bioimpedance and pulse applanation tonometry. We derive and evaluate multiple parameters regarding their correlation to reference blood pressure. These stem from features of the resulting warping paths of dynamic and derivative dynamic time warping. The warping is performed between adjacent pulses or to a reference waveform. Spearman Rho coefficients of up to 0.98 and averaging 0.77 at highly significant p-values are recorded for single parameters. The mean absolute deviation reaches 0.08 . The results indicate there are negligible lags between reference and parameter curves. The sign of the correlation coefficients is consistent only for a small subset of parameters; the underlying cause could not yet be identified. We conclude that the warping path approach seems a promising way to go, yet still needs refinement. In particular, developing a time and amplitude warping method is paramount. Since warping quantizes all the morphological changes in the pulse wave without fiducial point detection, it could become a powerful tool for future investigations. 


\section{Using singular value decomposition for generalized linear autoregression of signals}

Thomas Schanze, IBMT, FB Life Science Engineering (LSE), Technische Hochschule Mittelhessen (THM), 35390 Gießen, Germany, thomas.schanze@1se.thm.de

Autoregressive models (AR) are fundamental for analysis, representation, and prediction of signals. AR modelling uses the premise that past signal values influence current ones. This influence is causal and is modelled as a linear superposition, because a weighted addition of past values is used. The calculation of the required linear superposition parameters or weights can be done by the classical Yule-Walker approach or by least squares procedures. Here we show how to use singular value decomposition (SVD) for generalized linear autoregression (GLAR), i.e. using SVD to compute the weights of a linear combination of functions of given signal values and to check or optimize the GLAR model. The GLAR approach opens the possibility to take directly into account non-linear influences from past to current signal values. The potential of this approach for analysis and representation is presented and demonstrated for simulated signals, i.e. pure and noisy sequences of non-linear recursions, and biomedical signals. 


\section{Multimodal Sensor Fusion for Robust Heart Rate Variability Analysis in the Frequency Domain}

Timo Tigges, Chair of Electronics and Medical Signal Processing, Technische Universität Berlin,Berlin, Germany, timo.tigges@tu-berlin.de

Thomas Büchler, Chair of Electronics and Medical Signal Processing, Technische Universität Berlin, Berlin, Germany Alexandru Pielmus, Chair of Electronics and Medical Signal Processing, Technische Universität Berlin, Berlin, Germany

Michael Klum, Chair of Electronics and Medical Signal Processing, Technische Universität Berlin, Berlin, Germany

Aarne Feldheiser, Charité University Medicine Berlin, Campus Virchow-Klinikum, Department of Anaesthesiology and Intensive Care Medicine, Berlin, Germany

Oliver Hunsicker, Charité University Medicine Berlin, Campus Virchow-Klinikum, Department of Anaesthesiology and Intensive Care Medicine, Berlin, Germany

Reinhold Orglmeister, Chair of Electronics and Medical Signal Processing, Technische Universität Berlin, Berlin, Germany

Heart rate variability (HRV) analysis is a valuable tool in the investigation of cardiovascular regulation by the autonomic nervous system. In clinical care, generally, HRV parameters are calculated based on tachograms that are derived from ECG recordings. In case the ECG signal is disturbed by artifacts or exhibits intervals without valid recordings, it appears beneficial to exploit other biosignals that also contain information on cardiac cycles like for example photoplethysmograms (PPG). In this work, we present a novel approach for the fusion of multimodal biosignal recordings for a robust HRV estimation in the frequency domain while adhering to the individual measurement uncertainties of the fused signals.

The proposed method is based on the generalised Lomb-Scargle periodogram estimation scheme, which was extended to handle multiple noisy observations of a mutual process. As a basis for the evaluation of this method, we recorded a dataset consisting of an ECG and two PPG (fingertip and in-ear) signals of 5-minute duration. These signals were recorded from 28 healthy subjects (19 male, 9 female) under resting conditions. To estimate the signal inherent measurement uncertainties, we evaluated the beat-to-beat heart period differences from the ECG reference to the two PPGderived pulses. Then, we gradually removed more signal intervals from the ECG-based tachogram and assessed at what ECG data coverage fraction the frequency domain HRV parameters (VLF, LF, HF) from our fusion approach are more precise than the fragmented ECG signal. The fully covered ECG signal was used as reference.

The estimated measurement uncertainty for the fingertip PPG was $5.12 \mathrm{~ms}$ where the in-ear PPG resulted in a noise variance of $16.88 \mathrm{~ms}$. For the parameters VLF and LF, the fusion approach proved already to be beneficial at as little as $5 \%$ of missing ECG signal. The estimation of the high frequent parameter HF was superiorly estimated by our proposed method after $27 \%$ of missing ECG data. 


\section{Distribution of dominant mapping electrodes along splines of mini basket cathe- ters for distinct segments within the left atrium}

Laura Anna Unger, Institute of Biomedical Engineering (IBT), Karlsruhe Institute of Technology (KIT), Karlsruhe, Germany, laura.unger@kit.edu

Tobias Georg Oesterlein, was with Institute of Biomedical Engineering (IBT), Karlsruhe Institute of Technology (KIT), Karlsruhe, Germany; is now with Boston Scientific, Marlborough, MA, USA, tobias.oesterlein@bsci.com Armin Luik, Städtisches Klinikum Karlsruhe, Karlsruhe, Germany, Armin.Luik@klinikum-karlsruhe.de Claus Schmitt, Städtisches Klinikum Karlsruhe, Karlsruhe, Germany, Claus.Schmitt@klinikum-karlsruhe.de Olaf Dössel, Institute of Biomedical Engineering (IBT), Karlsruhe Institute of Technology (KIT), Karlsruhe, Germany, olaf.doessel@kit.edu

Three-dimensional electroanatomical mapping systems have become a standard tool to assist electrophysiologists in the diagnosis and treatment of atrial tachycardia. Distributors deploy various mapping catheters with an increasing number of electrodes such as the IntellaMap mini basket catheter Orion ${ }^{\mathrm{TM}}$ (Boston Scientific). The Orion ${ }^{\mathrm{TM}}$ catheter allows for electrogram acquisition without being in specific alignment with the endocardium due to equidistantly distributed electrodes on eight rotationally symmetric splines. Only a fraction of its 64 electrodes is in appropriate wall contact for each mapping position. In this study, we investigated the distribution of mapping electrodes with appropriate wall contact along splines for distinct left atrial segments.

Five subjects presenting with atrial flutter underwent an electrophysiological study with the Orion ${ }^{\mathrm{TM}}$ catheter. The left $^{\mathrm{T}}$ atrial anatomies were segmented patient-specifically including segments for the anterior, posterior, and lateral wall, the left and right pulmonary veins, the appendage, the roof, and the septum. We analyzed mapping data during stable catheter positions of at least $5 \mathrm{~s}$ and a minimum of 6 electrodes in contact with the endocardium. The quality of wall contact was determined by an electrode's distance to the endocardium with an upper limit of $5 \mathrm{~mm}$. In the septal segment, proximal electrodes tended to show better wall contact than in any other anatomical region. The wall contact within the pulmonary vein segments and the appendage decreased from equatorial to proximal electrodes. A decrease in wall contact from distal to proximal electrodes was notable in the remaining segments being most prominent for the roof and the lateral wall.

In conclusion, we showed that all electrodes contribute to the mapping procedure in the left atrium with the proximal electrodes being of least and the distal electrodes of highest significance for most anatomical segments. Our findings could help to determine starting points for future improvements in catheter design. 


\section{Statistische Beschreibung der Druckverteilung zur Beurteilung der Beatmung}

Lisa Steinbrecher, Technische Hochschule Mittelhessen, Germany, lisa.steinbrecher@ges.thm.de Keywan Sohrabi, Technische Hochschule Mittelhessen, Germany, keywan.sohrabi@ges.thm.de Andreas Weissflog, ThoraTech GmbH, Germany, weissflog@thoratech.de Florian Schudt, Technische Hochschule Mittelhessen - University of Applied Sciences, Germany, florian.schudt@mni.thm.de

Henning Schneider, Technische Hochschule Mittelhessen - University of Applied Sciences, Germany, henning.schneider@mni.thm.de

Werner Seeger, Universitätsklinikum Giessen, Germany, Werner.Seeger@innere.med.uni-giessen.de Volker Gross, Technische Hochschule Mittelhessen, Germany, volker.gross@ges.thm.de

Die mechanische Beatmung eines Patienten dient der Unterstützung oder dem Ersatz der Spontanatmung, die unzureichend oder nicht vorhanden ist. Der Erfolg der Beatmung, wird im klinischen Alltag auf Grund der arteriellen Blut-Gas-Analyse (BGA) beurteilt. Die individuelle Anpassung der Beatmungsparameter erfolgt also nach dem „trial and error“ - Prinzip. Eine Aufzeichnung der patientenseitigen Beatmungsparameter würde einen genaueren Überblick der Effizienz der Beatmung liefern. Ein Patient kann innerhalb eines Tages unterschiedliche Beatmungsmuster haben, die unterschiedliche Einstellungen erfordern. Je nach zu behandelnder Krankheit können die Unterschiede zwischen Patienten sehr groß sein, sodass generelle Aussagen zur Beatmungsmethodik bei einigen Krankheiten nicht möglich sind. Das Ziel dieser Arbeit ist die Entwicklung und Beschreibung weiterer Parameter, die einen schnelleren Überblick zeitabhängiger Darstellungen ermöglichen und die Beurteilung der Beatmungsakzeptanz numerisch stützen. Parameter die, die Ausprägungen der Peaks der Werteverteilungen und somit die Stabilität der Messung wiederspiegeln wurden berechnet. Diese Parameter sind der charakteristische Wert (CV), der das Verhältnis von Peak-Breite bei 70\% der Höhe zur Peak-Höhe ist und der trianguläre Index (TI), der das Verhältnis von Peak-Fläche zur Peak-Höhe ist. Beispiele für eine stabile, instabile und eine nicht-identifizierbare Messung sind gegeben. Grenzwerte zur Klassifizierung einzelner Messungen anhand der Werte sind noch zu definiert. 


\section{Causal assessment of the central-cardiovascular network pathways in a neuro- logical disorder}

Steffen Schulz, Institute of Innovative Health Technologies IGHT, University of Applied Sciences, Jena, Germany, Steffen.Schulz@eah-jena.de

Aniol Serra Juhé, Department of Automatic Control (ESAII), Universitat Politècnica de Catalunya, Barcelona, Spain, aniolserra@hotmail.com

Beatriz Giraldo, Department of Automatic Control (ESAII), Universitat Politècnica de Catalunya, Barcelona, Spain, beatriz.giraldo@upc.edu

Jena Haueisen, Institute of Biomedical Engineering and Informatics, University of Technology, Ilmenau, Germany, jens.haueisen@tu-ilmenau.de

Karl-Jürgen Bär, Department of Psychiatry and Psychotherapy, University Hospital, Jena, Germany, KARL-JUERGEN.Baer@med.uni-jena.de

Andreas Voss, Institute of Innovative Health Technologies IGHT, University of Applied Sciences, Jena, Germany, andreas.voss@eah-jena.de

Network physiology aims to define healthy and diseased states by analysing structural, dynamical and regulatory alterations in the interaction of physiological systems and sub-systems.

In this study, we investigated the central-cardiovascular network applying linear and nonlinear causal coupling approaches, the normalized short-time partial directed coherence (NSTPDC) and multivariate transfer entropy (MuTE) in 17 patients suffering from paranoid schizophrenia (SZO) and 17 healthy subjects (CON). From all participants, continuous heart rate (successive beat-to-beat intervals, BBI), non-invasive continuous systolic blood pressure (successive sys-

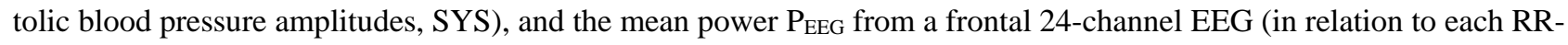
interval) were recorded for 15 minutes under resting conditions.

We found highly significantly reduced linear as well as nonlinear central-cardiovascular couplings in SZO compared to CON. The nonlinear influences (MuTE) from BBI and SYS to $\mathrm{P}_{\mathrm{EEG}}$ as well as from $\mathrm{P}_{\mathrm{EEG}}$ to BBI and SYS were reduced in SZO compared to CON. The linear influence (NSTPDC) from SYS to $\mathrm{P}_{\mathrm{EEG}}$ was stronger in CON than in SZO, whereas the linear influence from $\mathrm{P}_{\mathrm{EEG}}$ to SYS was higher in SZO than in CON.

The central-cardiac coupling $\left(\mathrm{P}_{\mathrm{EEG}}-\mathrm{BBI}\right)$ seems to be of nonlinear nature and is significantly reduced in each direction in SZO. The central-vascular coupling ( $\mathrm{P}_{\mathrm{EEG}}-\mathrm{SYS}$ ) was more pronounced than the nonlinear one in SZO and strongly focused on maintaining the blood pressure regulation (baroreflex loop).

For SZO, the central part of this closed-loop seems to influence more strongly the autonomic system than the autonomic system the central one. Particularly the central nerve system stronger controlles the vascular (SYS) and less the cardiac (BBI) system.

This study provides a more in-depth understanding of the interplay of neuronal and autonomic cardiovascular regulatory pathways in the neurological disorder schizophrenia, as well as a greater insight into the complex central autonomic network. 